\title{
Distribution Network Reconfiguration Approach of Avoiding Infeasible Solutions
}

\author{
Juan WEN \\ School of College of Electrical and Information Engineering \\ Hunan University \\ Changsha, P. R. China \\ 1147498202@qq.com
}

\author{
Yanghong TAN \\ School of College of Electrical and Information Engineering \\ Hunan University \\ Changsha, P. R. China \\ tanyh@126.com
}

\begin{abstract}
A novel distribution network reconfiguration approach of avoiding infeasible solutions is proposed based on breaking up the whole into parts strategy. In which, the solution space is divided into several subspaces which eliminate the unfeasible trial candidates and reduce search space. An improved quantum particle optimization algorithm is applied in each subspace solution to search a good solution set. Then the coordination agent would select a solution from each subspace solution set, and coordinate them until all the operational constraints are satisfied. To validate the proposed reconfiguration approach, computer simulations are conducted on distribution systems. The obtained results are compared with other methods available in the previous works.
\end{abstract}

Keywords: distribution network reconfiguration; breaking up the whole into parts strategy; improved particle swarm optimization

\section{INTRODUCTION}

Distribution network reconfiguration is the process of altering the network structure by changing the status of switches [1]. This is a complicated combinatorial optimization problem which may be subjected to one or many objectives as satisfying the operating constrains. Recently several algorithms based on artificial intelligence techniques are used to solve this optimization problem. A good coding scheme of the status variable plays a key role in the process of intelligence algorithms, since it indicates whether or not a large number of infeasible trial solutions would appear [2-3].

The existing techniques of handling the infeasible solutions have been implemented from various angles. An algorithm based on branch-to-node incidence matrix is presented to check the candidate solutions [4]. G.A. Carcamo has proposed a heuristic graph compression approach to reduce the dimension of candidate solutions [5]. These approaches can judge the feasibility of each trial solution. However, the iteration numbers of obtaining an optimum solution may be increased in the reconfiguration algorithm. In reference [6], the switch position of each branch in the radial network topology is formed by the binary coding (closed/open) and the network topology string only stores the opened switches positions in reference [7]. Although the binary encoding method is easy to realize, the probability of radial trial solutions generated by intelligence algorithms may be low. Reference [8] has employed a real number encoding scheme based on "loop cut set" in which each chromosome is a list of indices of opened switches. A decimal encoding strategy is employed for particle swarm optimization algorithm. The special strategy reduces the encoding length and enhances the proportion of feasible topology configurations [9]. The above encoding approaches could

This work was supported by national natural science foundation of China (No.61102039) and Hunan provincial natural science foundation of China (No. 14JJ7029). decrease the number of infeasible solutions. But they require a specific test to validate the resulting topologies. If the topologies are not feasible, the procedure of generating trial solutions will need to be applied again. In reference [10], a matroid theory encoding which all trial individuals are claimed to be radial configurations is presented. This approach avoids tedious mesh checks for the topology constraint validation. For complex real distribution systems, which contain multiple cross loops, it may not be sufficient due to all feasible topology candidates are obtained.

This paper designs a breaking up the whole into parts encoding scheme to overcome the drawbacks in the previous encoding strategies. It is used to generate candidate solutions which are valid and feasible. Meanwhile, this scheme is applied to the quantum particle swarm optimization of distribution network reconfiguration. To verify the effectiveness of the encoding strategy, comparative studies are conducted on IEEE33 bus test system with encouraging results.

\section{BREAKING UP THE WHOLE INFO PARTS SCHEME}

Distribution system can be represented by a topology graph, whose nodes and branches are corresponding to bus bars and to electric elements, respectively. Due to some graph concepts, techniques, and theorems are broadly adopted in different research areas, it can be deal with the problem of network reconfiguration.

\section{A Distribution Network Simplified}

Consider a distribution system with a set of n nodes, that is, $G=(V, E)$, where $V=\left\{v_{1}, v_{2}, \ldots v_{n}\right\}$ is a set of labeled nodes and $E=\left\{e_{1}, e_{2}, \ldots e_{n}\right\}$ is a set of branches. We introduce a concept of adjacency matrix $A=\left(a_{i j}\right)_{n \times n}$, Where $n$ is the number of nodes, $a_{i j}$ is a binary variable, if $a_{i j}=1$ demonstrates $i$-node is connected to another $j$-node by an edge and 0 otherwise. Another concept of $i$-node degree is defined as $D_{v i}=\sum_{j=1}^{n} a_{i j}$. A distribution system incorporates a few kinds of nodes, i.e. power source nodes, terminal nodes, electric T-nodes and junction nodes. So the different set of degree value $D_{v i}=1, D_{v i}=2$ and $D_{v i}>3$ is corresponding to the terminal nodes, junction nodes and T-nodes. Power source nodes, terminal nodes and T-nodes are collectively called special nodes. The complex distribution network is simplified topology which incorporates special nodes and corresponding branch chains. Moreover, distribution feeders are connected in a network structure by sectionalizing switches and tie switches. If all the switches are closed, the feeders can be looped. The encoding strategy 
which only takes the loop switches representation into consideration as distribution systems operate with a radial topology. A diagrammatically representation of a distribution network is illustrated, using 33-bus system as shown in reference [10]. Fig.1 shows the simplified generation. Note that the number of nodes reduces from 33 to 8 . The new switch chains are expressed as L1 L12.

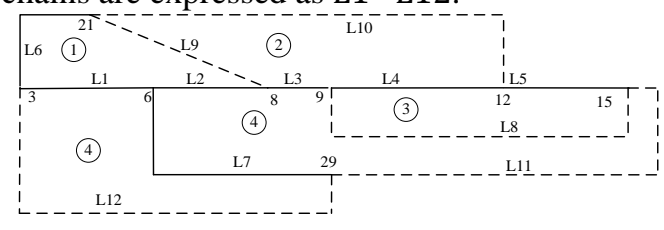

Fig.1 Simplified graph of 33-bus system

\section{B Breaking Up The Whole Into Parts Code Scheme}

Numeric reconfiguration algorithms are based on fundamental loops vectors. We introduce a novel method for determining the loops. It is required that there is minimum number of switches in each loop. A serial number is assigned to each loop. TABLE I describes loops segmentation of Fig.1.

TABLE I. LOOPS SEGMENTATION OF FIG.1

\begin{tabular}{|c|c|}
\hline loop number & switch chains \\
\hline (1) & L1 L2 L6 L9 \\
\hline (2) & L3 L4 L9 L10 \\
\hline (3) & L4 L5 L8 \\
\hline (4) & L1 L7 L12 \\
\hline 5 & L2 L3 L7 L8 L11 \\
\hline
\end{tabular}

The relationship between any two loops can be described as a loop-switch matrix which has one row for each loop and one column for each the set of switches. It is defined as:

$$
L=\left(l_{i j}\right)_{m \times m}=\left[\begin{array}{ccccc}
L 6 & L 9 & 0 & L 1 & L 2 \\
L 9 & L 10 & L 4 & 0 & L 3 \\
0 & L 4 & L 5 & 0 & L 8 \\
L 1 & 0 & 0 & L 12 & L 7 \\
L 2 & L 3 & L 8 & L 7 & L 1
\end{array}\right] 1
$$

Where $L$ is loop-switch matrix, $m$ is the number of loops, $l_{i j}$ is a variable, if $l_{i j}=L_{x}\left(L_{x} \in(L 1 \sim L 12)\right)$ demonstrates there is a public switch chain $L_{x}$ between $i$-loop and $j$-loop and $l_{i j}=0$ otherwise.

Obviously, $L$ is a symmetric matrix. The diagonal parameters represent that corresponding switches are only in one loop. We expect that the candidate topologies maintain the radiality of the system in the process of reconfiguration. To address this problem, the following criteria must be considered via analyzing the loop incidence matrix.

i. Number of opened switches is equal to the number of loops of a distribution system.

ii. Diagonal set of switch chains should be at least one open switch. It is important for avoiding loops.

iii. For avoiding the isolated nodes, only one switch is opened in the same set of switch chains.

iv. There must be at least one open switch in each row or column of $L$ matrix.

On the basis of these criteria, the breaking up the whole into parts scheme is established. In this strategy, each loop is considered as one control variable and one diagonal parameter of loop-switch matrix must be chosen. It means that the corresponding loop label of this parameter row label is fixed. Other opened switches are obtained by applying the previous criteria. Therefore, the scheme of no coding redundancy is described as TABLE II.

TABLE II. FEASIBLE TRIAL SOLUTIONS

\begin{tabular}{|c|c|}
\hline fixed loop number & form of solution \\
\hline 1 & {$[\{L 6\}\{L 9 L 10 \mathrm{~L} 4 \mathrm{~L} 3\}\{\mathrm{L} 5 \mathrm{~L} 8\}\{\mathrm{L} 1 \mathrm{~L} 12 \mathrm{~L} 7\}\{\mathrm{L} 2 \mathrm{~L} 11\}]$} \\
\hline$(2)$ & {$[\{\mathrm{L} 9 \mathrm{~L} 1 \mathrm{~L} 2\}\{\mathrm{L} 10\}\{\mathrm{L} 4 \mathrm{~L} 5 \mathrm{~L} 8\}\{\mathrm{L} 12 \mathrm{~L} 7\}\{\mathrm{L} 3 \mathrm{~L} 11\}]$} \\
\hline$(3)$ & {$[\{\mathrm{L} 9 \mathrm{~L} 1 \mathrm{~L} 2\}\{\mathrm{L} 4 \mathrm{~L} 3\}\{\mathrm{L} 5\}\{\mathrm{L} 12 \mathrm{~L} 7\}\{\mathrm{L} 8 \mathrm{~L} 11\}]$} \\
\hline 4 & {$[\{\mathrm{L} 9 \mathrm{~L} 1 \mathrm{~L} 2\}\{\mathrm{L} 4 \mathrm{~L} 3\}\{\mathrm{L} 8\}\{\mathrm{L} 12\}\{\mathrm{L} 7 \mathrm{~L} 11\}]$} \\
\hline 5 & {$[\{\mathrm{L} 9 \mathrm{~L} 1 \mathrm{~L} 2\}\{\mathrm{L} 4 \mathrm{~L} 3\}\{\mathrm{L} 8\}\{\mathrm{L} 7\}\{\mathrm{L} 11\}]$} \\
\hline
\end{tabular}

\section{SOLUTION METHOD}

Particle swarm optimization technique has recently attracted much attention as it is a powerful way to reach the global optimums. This paper proposes the improved quantum particle swarm optimization (QPSO) for avoiding infeasible solutions.

\section{A Reconstruction Mathematical Formulation}

The distribution system reconfiguration can be described as a nonlinear mixed optimization problem by turning on/off switches with the objective of minimizing active power losses for satisfying a group of constraints. The mathematical formulation can be expressed as follows:

$$
\min f_{\text {ploss }}=\sum_{i=1}^{n} \sum_{j=1}^{n} r_{i j} \frac{\left|S_{i j}\right|^{2}}{V_{i}^{2}}
$$

The constraints include current thermal, transmission power capacity, radiation-shaped network topology, and node voltage magnitude constraints [10].

\section{B Improved Quantum Particle Swarm Optimization}

The improved quantum particle swarm algorithm (IQPSO) is based on the typical QPSO. A possible solution is represented as the coordinates of each particle. Particles move through the $D$-dimensional searching space using a combination of an attraction to the optimal solution. They have a fitness value evaluated to find the best solution according to their own and their companion's searching experience. A vector $x_{i d}=\left(x_{i 1}, x_{i 2} \ldots x_{i D}\right)$ represents the position of a particle in D-dimensional search space. The updated position of particle $i$ is modified under the following equation that calculates $t+1$ iteration position $x_{i d}(t+1)$ based on its previous position $x_{i d}(t)$.

$$
x_{\text {id }}(t+1)=\operatorname{round}\left(p_{\text {id }}(t) \pm \beta * \mid \text { mbest }(t)-x_{\text {id }}(t) \mid * \ln \left(\frac{1}{u}\right)\right)
$$

Where $x_{i d}(t)$ and $x_{i d}(t+1)$ are the original position and new position of $i$-particle, respectively. $p_{i d}(t)$ represents the current best position of particle i. $\operatorname{mbest}(t)$ is the average best position of all particles. The integral function uses round (). $\beta$ is convergence coefficient. 
C Constructed IQPSO Based On Breaking Up The Whole Into Parts Code Scheme

A good code scheme plays a critical role for IQPSO as it can extremely restrict unfeasible trial solutions appearing at each generation in distribution system reconfiguration. In this paper, we use the code scheme of section II to avoid them.

The relationship between the switches and the decimal number are shown in Fig.1. Each loop represents a variable. The search pace is divided into multiple subspaces according to the fixed loop label. According to the TABLE II, the loop combination switches of a subspace are described as TABLE III.

\section{TABLE III. LOOP CONSTRUCTED SWITCHES}

\begin{tabular}{|c|c|c|c|}
\hline $\begin{array}{c}\text { Fixed loop } \\
\text { number }\end{array}$ & $\begin{array}{c}\text { Loop } \\
\text { number }\end{array}$ & Constructed switches & $\begin{array}{l}\text { Decimal } \\
\text { encoding }\end{array}$ \\
\hline \multirow{5}{*}{ (1) } & (1) & $2,18,19,20$ & $1-4$ \\
\hline & (2) & $8,9,10,11,21,33,35$ & $1-7$ \\
\hline & (3) & $12,13,14,34$ & $1-4$ \\
\hline & (4) & $3,4,5,22,23,24,25,26,27,28,37$ & $1-10$ \\
\hline & (5) & $6,7,15,16,17,29,30,31,32,36$ & $1-9$ \\
\hline \multirow{5}{*}{ (2) } & (1) & $3,4,5,6,7,33$ & $1-6$ \\
\hline & (2) & 21,35 & $1-2$ \\
\hline & (3) & $9,10,11,12,13,14,34$ & $1-7$ \\
\hline & (4) & $22,23,24,25,26,27,28,37$ & $1-8$ \\
\hline & (5) & $8,15,16,17,29,30,31,32,36$ & $1-9$ \\
\hline \multirow{5}{*}{ (3) } & (1) & $3,4,5,6,7,33$ & $1-6$ \\
\hline & (2) & $8,9,10,11$ & $1-4$ \\
\hline & (3) & $12,13,14$ & $1-3$ \\
\hline & (4) & $22,23,24,25,26,27,28,37$ & $1-8$ \\
\hline & (5) & $15,16,17,29,30,31,32,36,34$ & $1-9$ \\
\hline \multirow{5}{*}{ (4) } & (1) & $3,4,5,6,7,33$ & $1-6$ \\
\hline & (2) & $8,9,10,11$ & $1-4$ \\
\hline & (3) & 34 & 1 \\
\hline & (4) & $22,23,24,37$ & $1-4$ \\
\hline & (5) & $15,16,17,29,30,31,32,36,25,26,27,2,8$ & $1-12$ \\
\hline \multirow{5}{*}{ (5) } & (1) & $3,4,5,6,7,33$ & $1-6$ \\
\hline & (2) & $8,9,10,11$ & $1-4$ \\
\hline & (3) & 34 & 1 \\
\hline & (4) & $25,26,27,28$ & $1-4$ \\
\hline & (5) & $15,16,17,29,30,31,32,36$ & $1-8$ \\
\hline
\end{tabular}

The searching space of the system is divided into five subspaces. Each row represents candidate switches of a dimension particle in each subspace. A set of solutions is formed while a random switch is extracted from each line of the TABLE III. Moreover, all the loops switches of each subspace are not duplicated. It means that these trial solutions are validity and non-redundancy. This paper adopts multi-dimension strategy to prevent the escape of each particle. Equations (4) and (5) mean that each particle deviations of minimum limit and maximum limit.

$$
\begin{aligned}
\operatorname{Lix} & =\left[\begin{array}{llll}
\operatorname{Lix}_{1} & \operatorname{Lix}_{2} & \ldots & \mathrm{Lix}_{n}
\end{array}\right] \\
\operatorname{Uix} & =\left[\begin{array}{lllll}
\operatorname{Uix}_{1} & \mathrm{Uix}_{2} & \ldots & \mathrm{Uix}
\end{array}\right]
\end{aligned}
$$

Where $U_{i x}$ and $L_{i x}$ are the minimum and maximum limit matrix of particles in each subspace. $n$ is the number of dimensions. For example, if loop (1) is fixed in table 4, we set $L_{i x}=\left[\begin{array}{lllll}1 & 1 & 1 & 1 & 1\end{array}\right]$ and $U_{i x}=\left[\begin{array}{lllll}4 & 7 & 4 & 1 & 0\end{array}\right]$. If the loop (2), (3), (4) or (5) is fixed, the algorithm will do the similar procedure as the above section for generating others feasible solutions, $U_{i x}$ and $L_{i x}$, respectively.

\section{Implementation Procedure for Reconfiguration Problems}

The flow of IQPSO algorithm is described as follows:

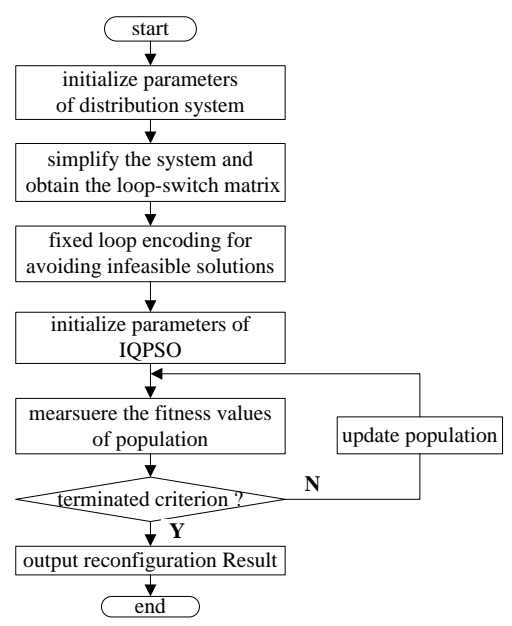

Fig.3 Flow chart of the IQPSO algorithm

\section{CASE STUDIES}

The proposed method of avoiding infeasible solutions is applied to 33-bus radial distribution system [10]. The substation voltage is considered as 1 p.u., and all sectionalizing and tie switches are candidate switches for reconfiguration. The algorithm program was implemented using MATLAB, and the simulations were done on a computer with Intel core IV, 3.0 GHz, 2GB RAM.

TABLE IV shows the combination number of trial solutions using binary coding [5], improved binary coding [6] and decimal encoding [8].

TABLE IV. SEARCH SPACE OF DIFFERENT ENCODING

\begin{tabular}{|l|l|}
\hline encoding & Resultant search space \\
\hline binary coding & $2^{37}=1.3744 * 10^{11}$ \\
\hline improved binary coding & $C_{37}^{5}=435897$ \\
\hline decimal encoding & $10 \times 15 \times 7 \times 21 \times 11=242550$ \\
\hline Proposed encoding method & 23232 \\
\hline
\end{tabular}

The dimension of vector equals the number of system branches while the binary coding is used to code 33-bus system and the combination number is $2^{37}$. If this method is applied to reconfiguration algorithms, a population operation is likely to makes infeasible individuals. The element of improved binary coding vector shows the order of the open switch numbers. Therefore, the number of combination and infeasible solutions are reduced drastically. The decimal encoding illustrates each fundamental loop should be made to have fewer switches to minimize the amount of searching space needed. Comparing binary coding, only have 242550 combination number and the infeasible solutions ratio is reduce to $74.38 \%$. The fixed loop encoding generates 23232 feasible solutions. It is obviously that avoids the drawbacks of other methods.

In the algorithm of IQPSO, the number of individuals is 50 and maximum iterations set 100 . The maximum and minimum of weighting values are 0.9 and 0.4 , respectively. The upper limit and lower limit of particles are set as the total 
number of loop constructed switches by TABLE III. The simulation results summarized and compared to methods presents in [5], [6] and [8].

\section{TABLE V. CONFIGURATION RESULTS}

\begin{tabular}{|c|c|c|c|}
\hline method & Final configuration & $p_{\text {loss }}$ & $\begin{array}{c}\text { Voltage profile } \\
\text { (p.u.) }\end{array}$ \\
\hline Initial system & {$\left[\begin{array}{llllll}33 & 34 & 35 & 36 & 37\end{array}\right]$} & 202.4895 & $0.9134 \sim 1$ \\
\hline [5] & {$\left[\begin{array}{lllll}7 & 14 & 9 & 32 & 37\end{array}\right]$} & 139.530 & $0.9411 \sim 1$ \\
\hline [6] & {$\left[\begin{array}{llllll}7 & 14 & 9 & 32 & 37\end{array}\right]$} & 139.550 & $0.9410 \sim 1$ \\
\hline [8] & {$\left[\begin{array}{llllll}7 & 14 & 9 & 32 & 37\end{array}\right]$} & 139.4410 & $0.9413 \sim 1$ \\
\hline IQPSO & {$\left[\begin{array}{llllll}7 & 14 & 9 & 32 & 37\end{array}\right]$} & 139.4410 & $0.9413 \sim 1$ \\
\hline
\end{tabular}

TABLE $\mathrm{V}$ represents the effectiveness of proposed method which could have achieved the optimal configuration compared to other methods. The real power loss after reconfiguration is reduced by $31.19 \%$ of initial system. The minimum bus voltage also is raised from $0.9131 \mathrm{p}$. u. to $0.9413 \mathrm{p}$. u. These results are identical to those obtained by BPSO [5] genetic algorithm [6] and QPSO [8]. Fig 4 shows the voltage profile improvement achieved by IQPSO. As shown, most of the bus voltages have been greatly improved after network reconfiguration.

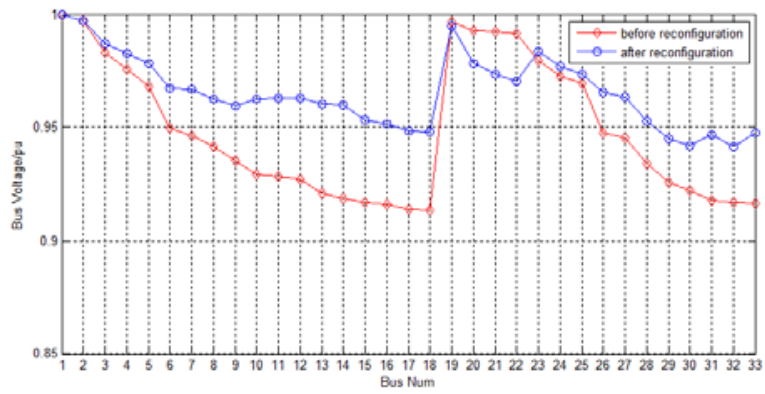

Fig.4 Voltage profile for the 33-bus system after using IQPSO

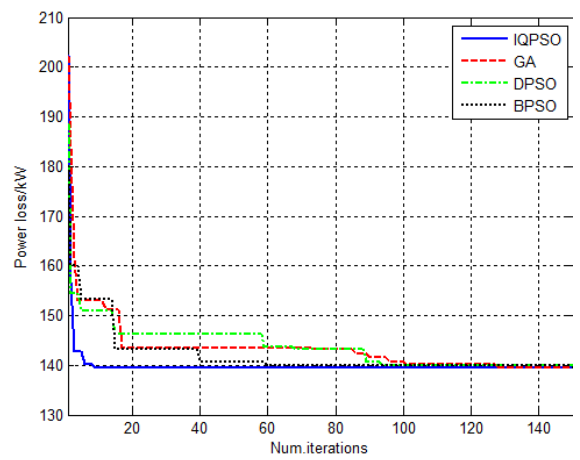

Fig 5 Convergence rate

To evaluate the performance of the proposed approach, this case is repeatedly solved. Fig 5 depicts the convergence rate of IQPSO for best cases of the 50 independent runs. The best for these 50 runs compared with the best fitness values obtained by BPSO, GA and QPSO. The proposed IQPSO could find the global optimum point in 9th iteration and has a relatively fast convergence performance compared with the other algorithms.

\section{CONCLUSION}

In this paper, an improved quantum particle swarm optimization (IQPSO) is successfully applied to optimize radial network reconfiguration with objectives of improving the voltage profile and minimizing real power loss. A breaking up the whole into parts scheme designed, which can ensure each trial solution to be feasible in distribution system reconfiguration, has been presented to improve the efficiency of search process. IQPSO algorithm is introduced using this encoding scheme. It can converge to a satisfactory result with low number of Iterations. Simulation results are compared with other methods BPSO, GA, and DPSO available in the reference. The breaking up the whole into parts encoding scheme avoids infeasible solution and reduce search space significantly. The IQPSO algorithm outperforms the other reconfiguration methods in terms of computational speed and memory requirements, as it requires only analyze feasible solutions in each subspace. This method is useful for analyzing existing systems, helps in planning a future system.

\section{References}

[1] W. J. Cheng, C. H. Dong and G. R. Darling, “An Efficient Algorithm for Real-Time Network Reconfiguration in Large Scale Unbalanced Distribution Systems,” IEEE Tran on Power Systems, vol.11, no. 1, pp. 511-517, Feb, 1996.

[2] H. Salazar, R. Gallego and R. Romero, “Artificial neural networks and clustering techniques applied in the reconfiguration of distribution systems,” IEEE Transactions on Power Delivery, vol. 21, no. 3, pp. 1735 - 1742, Apr. 2006.

[3] B. Radha, R.T. F. Ah King and H. C. S. Rughooputh, "A modified genetic algorithm for optimal electrical distribution network reconfiguration," IEEE Congress on Evolutionary Computation, vol. 2, pp. 1472 - 1479, Apr. 2003.

[4] A. Y. Abdelaziz, F. M. Mohammed, S. F. Mekhamer, et al. "A Modified Particle Swarm Algorithm for Distribution Systems Reconfiguration," Electric Power Systems Research, vol. 79, no. 12, pp. 1521-1530, Apr. 2009.

[10] G. A. Carcamo, S. J. Garcia and J. E. Pezoa. "Greedy reconfiguration algorithms for Medium-Voltage distribution networks," IEEE Transactions on Power Delivery, vol. 24, no. 1, pp. 328-337, Jan. 2009.

[5] H. Hosseini, S. Jalilzadeh, V. Nabaei, et al. "Enhancing Deregulated Distribution Network Reliability for Minimizing Penalty Cost Based on Reconfiguration Using BPSO,” IEEE Power and Energy Conference, pp. 983-987, Johor Baharu, Malaysia, Dec. 2008.

[6] J. Z. Zhu, "Optimal reconfiguration of electrical distribution network using the refined genetic algorithm,” Elect. Power Syst. Res., vol. 62, pp. 37-42, 2002.

[7] R. T. F. A. King, B. Radha, and H. C. S. Rughooputh, “A real-parameter genetic algorithm for optimal network reconfiguration,” IEEE Int. Conf. Industrial Technology, vol. 1, pp. 54-59, Dec. 2003.

[8] F. Batrinu, E. Carpaneto and G. Chicco, "A Novel Particle Swarm Method for Distribution System Optimal Reconfiguration,” IEEE Power Tech, pp. 948-954, Russia, May, 2005.

[9] B. Enacheanu, B. Raison, R. Caire, et al. "Radial Network Reconfiguration Using Genetic Algorithm Based on the Matroid Theory," IEEE Tran on Power Systems, Vol. 23, No. 1, pp. 186-195, Feb. 2008.

[10] C. C. Chieh, T. M. Shen, "Application of Novel Charged System Search With Real Number String for Distribution System Loss Minimization,” IEEE Trans on Power Systems, Vol. 28, No. 4, pp. 3600-3609, 2013.

[11] J. Sun, W. Fang, X. Wu, et al, “Quantum-Behaved Particle Swarm Optimization: Analysis of Individual Particle Behavior and Parameter Selection,” Evolutionary Computation, Vol. 20 , No. 3, pp. 349 - 393, 2012. 